\title{
Retraction Note: On the external field of an ideal toroid with arbitrary cross section
}

\section{H. M. Ymeri}

Published online: 23 October 2013

(C) Springer-Verlag Berlin Heidelberg 2013

Retraction to: Electrical Engineering (1997) 80 1-4

DOI 10.1007/BF01235662

This paper has been retracted because it is to a large extent identical to paper "Page CH (1971) External Field of an Ideal Toroid. American Journal of Physics 39(9): 1039". Unfortunately, the author could not be found for a statement.

The online version of the original article can be found under doi:10.1007/BF01235662. 